\title{
FORMATION OF PERSONALIZED DIGITAL MODELS BASED ON TOMOGRAPHIC DATA OF THE PATIENT
}

\author{
S. Cherebylo ${ }^{1}$, E. Ippolitov ${ }^{1}$, M. Novikov ${ }^{1}$ and V. Vnuk ${ }^{1}$ \\ ${ }^{1}$ Institute on Laser and Information Technologies RAS - \\ Branch of the Federal Scientific Research Centre "Crystallography and Photonics" of RAS, Shatura, Russia
}

Commission II, WG II/10

KEY WORDS: Spiral computed tomography, digital model, computer modeling, segmentation of tomographic data.

\begin{abstract}
:
The introduction of information technologies into the practice of healthcare significantly changes the methods of diagnosis and treatment, the forms of interaction of doctors with patients and colleagues, the organization of treatment and restoration of health. Modern digital medicine makes it possible to increase the availability, quality and efficiency of medical care.

The development of modern three-dimensional modeling and the introduction of a new generation of spiral computed tomographs have significantly expanded the possibilities of using these information technologies in reconstructive surgery. The performed study deals with the problems of forming the personalized digital models of anatomical structures according to patient's tomographic data. The principles of tomographic image segmentation are considered. A review and comparison of the specialized software for obtaining of digital models with the use of tomographic data is given. The evaluation of the functionality, speed and quality of the models is presented. Practical recommendations on the use of the software for creating digital models for medical applications are given. The issues of certification of personalized models and medical products are discussed.
\end{abstract}

\section{INTRODUCTION}

The introduction of information technologies into the practice of healthcare significantly changes the methods of diagnosis and treatment, the forms of interaction of doctors with patients and colleagues, the organization of treatment and restoration of health. Modern digital medicine makes it possible to increase the availability, quality and efficiency of medical care.

The development of modern three-dimensional modeling and the introduction of a new generation of spiral computed tomographs have significantly expanded the possibilities of using these information technologies in reconstructive surgery (Maureen, 2017). The specialized software allows you to quickly process the patient's tomographic data and create digital three-dimensional models of any defect or pathology zone.

Digital models are increasingly applied in the preparation and planning of surgical intervention in maxillofacial surgery, neck and spine surgery, thoracic surgery, orthopedics and neurosurgery. They can be directly employed to create the copies of pathologies and defects of a particular patient using additive technologies (Ippolitov, 2015). The personalized plastic copies of anatomical structures allow for effective preplanning of complex operations. Based on the digital models of defects or pathology zones of specific human diseases, it is possible to create unified databases for intelligent medical decision support systems (Mirzoev, 2018).

Recently, the methods of computer and magnetic resonance imaging are also actively used for paleontological studies. In digital paleontology, they allow visualization of various pathologies and anthropometric studies of mummified remains without destroying the original object (Davies, 2017). In modern science, three-dimensional models and reconstructions of historical artifacts are often used for non-invasive studies of the mummification process, establishing gender and age, obtaining anthropological characteristics, as well as for conducting forensic medical examinations of ancient mummified objects. (Zeshch, 2016; Vasiliev, 2019).

\subsection{Segmentation of tomographic data}

The main purpose of a tomographic examination is medical diagnosis, clarification of the diagnosis or tracking the results of treatment. It allows you to identify the presence of pathology and get additional information about it. In most cases, this is done by sequential visual analysis of the image of sections formed by a CT scanner. The formation of three-dimensional reconstruction of tomographic data with the help of specialized programs is currently, which can significantly improve the detection of pathologies and conduct preliminary planning of complex surgical operations.

However, creating a three-dimensional reconstruction requires solving a number of problems, since the structures of interest and the surrounding tissues have a similar density and do not differ well on tomographic images. In these cases, there is a need for such an operation as segmentation-software selection of structures of interest in the required boundaries, followed by their visualization, analysis and export as a digital model file (Trukhan, 2014, Guang-Ye, 2016).

The digital model opens up additional possibilities: preoperative modeling of high-precision templates and implants based on the tomographic data of a specific patient, production of the copies of defects and pathologies using additive technologies for further analysis and planning of the operation, $3 \mathrm{D}$ printing of biological tissues and matrices for direct defect repair.

\subsection{The basic tools of segmentation}

1. Cutting out a segmented structure using a polygon. The tool is applied to three-dimensional reconstruction. There are two 
options: to remove everything that falls into the selected area, or, conversely, to delete everything around the selected area.

2. Erasing or drawing the required image structures with a tool of the specified size in the form of a ball or cylinder. The advantage of this tool is its locality: you can always choose the size of the tool to remove just what is required. In addition, this tool can be used on two-dimensional reconstruction slices, which allows you to perform manual segmentation of the desired structure by slices.

3. Selecting related areas. To do this, we define the concept of the minimum thickness of the structure that makes the object connected, i.e. if two parts of the object are connected by some structure whose thickness is less than the specified value, then these two parts will not be considered connected.

4. Morphological operations mute increasing or reducing the volume of reconstruction, which allows you to increase or reduce the current segmentation by a given number of voxels. For the convenience of the user, the amount of extension/reduction is set in millimetres.

5. Segmented structures permit performing binary operations between them: subtraction, intersection, and union. Two segmented structures are involved in any of these operations. When subtracting from the visibility mask of the target structure, all voxels of the segmentation mask of the additional structure are subtracted, as a result, all visible voxels of the additional structure become invisible in the target structure. When intersecting in the target structure visibility mask, only voxels that are present simultaneously in the target and additional structure segmentation mask remain. When combined, all voxels of the segmentation mask of the additional structure are added to the target structure visibility mask.

A segmented structure is a stored segmentation result that can be edited and visualized independently of other parts of the reconstruction (Musatyan, 2018). On the basis of any segmented structure, you can build its digital model mute an approximation of the surface of the segmented structure with polygonal elements (triangles). This is one of the most important segmentation results, since exporting the segmentation results as a polygon model allows you to work with them in most three-dimensional modeling programs. The "marching cubes" algorithm is used to construct grids, which is both simple and most effective for this task (Figure 1).
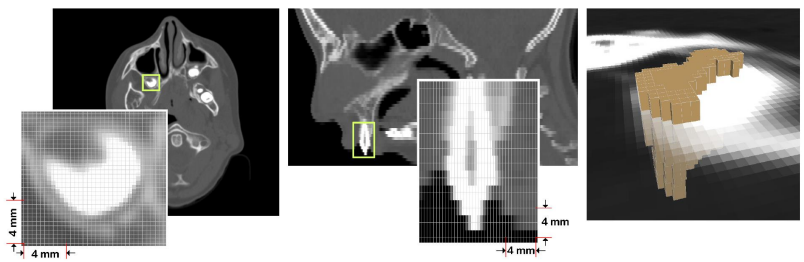

Figure 1. Tomographic image data: from pixels to voxels.

\section{PROGRAMS FOR PROCESSING AND CONVERTING DICOM IMAGES INTO A THREE- DIMENSIONAL MODEL}

\subsection{The software}

In this paper, several commercial and freely distributed software products were considered (Table 1).

\begin{tabular}{|l|l|l|l|}
\hline Software & \multicolumn{1}{|c|}{ Website } & License & Rating \\
\hline $\begin{array}{l}\text { Mimics } \\
\text { Medical }\end{array}$ & $\begin{array}{l}\text { www.materialise.com/ } \\
\text { Materialise, Belgium }\end{array}$ & paid & 9 \\
\hline $\begin{array}{l}\text { DICOM } \\
\text { Viewer }\end{array}$ & $\begin{array}{l}\text { https://inobitec.com/ } \\
\text { Inobitec, Russia }\end{array}$ & paid & 10 \\
\hline $\begin{array}{l}\text { 3D } \\
\text { Slicer }\end{array}$ & $\begin{array}{l}\text { https://www.slicer.org/ } \\
\text { team of specialists, USA }\end{array}$ & free & 7 \\
\hline $\begin{array}{l}\text { In } \\
\text { Vesalius }\end{array}$ & $\begin{array}{l}\text { www.cti.gov.br/en/invesal } \\
\text { ius free } \\
\text { Centro de Tecnologia da } \\
\text { Informação Renato } \\
\text { Archer, Brazil }\end{array}$ & 5 \\
\hline Drishti & $\begin{array}{l}\text { https://github.com/nci/dri } \\
\text { shti } \\
\text { National Computational } \\
\text { Infrastructure's VizLab, } \\
\text { Australia }\end{array}$ & free & 3 \\
\hline
\end{tabular}

Table 1. Software for processing and converting DICOM images into a three-dimensional model of the STL format.

Processing of two types of tomographic data to assess the performance was carried out on a computer with the following configuration: AMD Ryzen 71700 processor clock speed 3000 Hz, DDDR4 RAM 32 GB, Radeon RX 570 Series video card 4 GB.

\begin{tabular}{|l|c|c|c|c|}
\hline & \multicolumn{4}{|l|}{$\begin{array}{l}\text { CT of the head of 541 slices, } \\
\text { volume data 0.3 GB }\end{array}$} \\
\hline Software & \multicolumn{2}{|c|}{$\begin{array}{l}\text { CT data loading } \\
\text { time, c }\end{array}$} & \multicolumn{2}{l|}{$\begin{array}{l}\text { Time to build the } \\
\text { 3D model, c }\end{array}$} \\
\hline & load & process & voxel & stl \\
\hline $\begin{array}{l}\text { Mimics } \\
\text { Medical }\end{array}$ & 2 & 2 & 39 & 74 \\
\hline $\begin{array}{l}\text { DICOM } \\
\text { Viewer }\end{array}$ & 1 & 1 & 3 & 2 \\
\hline 3DSlicer & 39 & 24 & 24 & 6 \\
\hline $\begin{array}{l}\text { In } \\
\text { Vesalius }\end{array}$ & 14 & 7 & 88 & 10 \\
\hline Drishti & \multicolumn{2}{|l|}{54} & \multicolumn{3}{|c|}{187} \\
\hline
\end{tabular}

Table 2. Comparison of the time of processing and conversion of tomographic data. an easy task.

\begin{tabular}{|c|c|c|c|c|}
\hline \multirow{3}{*}{ Software } & \multicolumn{4}{|c|}{$\begin{array}{l}\text { CT of the body of } 5502 \text { slices, } \\
\text { volume data } 2.9 \mathrm{~GB}\end{array}$} \\
\hline & \multicolumn{2}{|c|}{$\begin{array}{l}\text { CT data loading } \\
\text { time, c }\end{array}$} & \multicolumn{2}{|c|}{$\begin{array}{l}\text { Time to build the } \\
\text { 3D model, c }\end{array}$} \\
\hline & load & process & voxel & stl \\
\hline $\begin{array}{l}\text { Mimics } \\
\text { Medical }\end{array}$ & 35 & 48 & 298 & 490 \\
\hline $\begin{array}{l}\text { DICOM } \\
\text { Viewer }\end{array}$ & 8 & 6 & 6 & 4 \\
\hline 3DSlicer & 492 & 221 & 135 & 25 \\
\hline $\begin{array}{l}\text { In } \\
\text { Vesalius }\end{array}$ & 486 & 113 & 196 & 27 \\
\hline Drishti & & - & & - \\
\hline
\end{tabular}

Table 3. Comparison of the time of processing and conversion of tomographic data, a difficult task. 


\subsection{A brief overview of the software}

A brief description of the features of the software.

1. Mimics Medical. Excellent functionality, well-designed interface, extensive image segmentation capabilities, high license cost.

2. DICOM Viewer. Good functionality, well-designed interface, extensive image segmentation capabilities, convenient operation with the voxel model, high performance, affordable license cost. 3DSlicer. Sufficient functionality, modular interface, sufficient image segmentation capabilities, many ready-made configurations for specialized tasks, free license.

3. InVesalius. Good opportunities for entry-level, modular interface, insufficient possibilities for segmentation of images with free license.

4. Drishti. Separate reading and processing programs, problems with reading DICOM format data, modular interface, insufficient image segmentation capabilities, free license. Figure 2 shows the images of models in STL format.
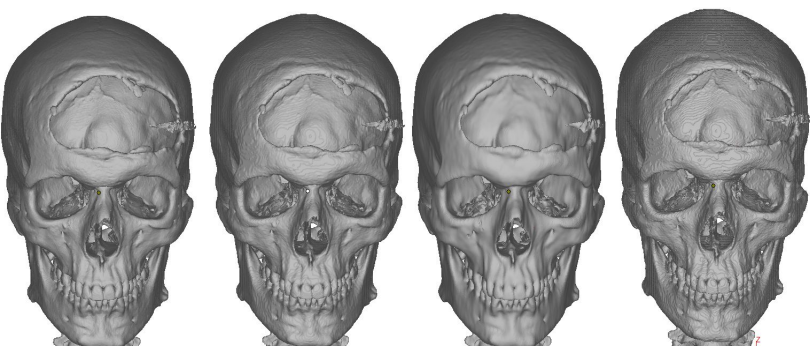

Fig. 2. Examples of models based on tomographic data, from left to right the results of the programs Mimics, DICOM Viewer, 3DSlicer, InVesalius.

\section{THE MAIN DIFFICULTIES OF SEGMENTATION}

We have been working on building the three-dimensional models based on CT data for 20 years since 2000 .

The quality of the image obtained in computed tomography is determined by five main factors: spatial resolution, contrast, noise and spatial uniformity, linearity and the presence of artifacts.

Spatial resolution is used to express the degree of image spotting and characterizes the ability to see an object of a certain density in an area containing substances with different densities. It depends on the collimation system, the size of the detector, the selected pixel size, and the size of the focal spot of the tube.

Image contrast resolution - the ability of the scanner to show small changes in the contrast of the tissues of large objects, is limited by noise that has a granular appearance.

Noise and spatial uniformity have different CT numbers for a fabric with the same density. They are mainly related to the effects of photons passing through the fabric. Depending on the source of occurrence, the noise is divided into quantum (the result of limiting the photons reaching the sensors), electronic (caused by the electrical interaction in the system itself), computational (approximations used in the measurement process) and radiation (caused by radiation scattering).
Image artifacts in computed tomography present any discrepancy between the CT numbers of the reconstructed image and the true attenuation coefficients of the object. The technology of image reconstruction is such that the measurements on all detectors are summed up, so any measurement errors appear in the images. Reconstruction errors can be caused by both a lack of data and the presence of various noises. Artifacts can manifest themselves as bands (error in a single measurement), darkening (gradual deviation of a group of channels), rings (calibration errors of a single detector), and distortion (spiral reconstruction).

Quite often, the objects with a high density, such as those made of metal, are present in the study area, which causes the artifacts in the form of stripes to appear in the image. This is because the density of the metal is outside the normal range of values that the tomograph can display, giving incomplete attenuation profiles.

The patient's movement also produces artifacts in the form of streaks or blurring of the image. This is because image reconstruction usually relies on the computer's ability to place the attenuation coefficient values in a matrix of pixels that has rows and columns. If there is movement during the scan, the computer cannot place the measured values in the correct spatial cell, which leads to image distortion.

All the noted problems associated with obtaining highly informative data in computed tomography require the use of special programs for processing halftone images before converting them into computer models corresponding to real biological objects. Various processing methods and techniques are used for this purpose.

This approach requires painstaking work and considerable time, since even small objects require manual processing of dozens of tomogram images.

To eliminate noise in the tomographic images, the function of selecting related areas is used (Fig. 3).
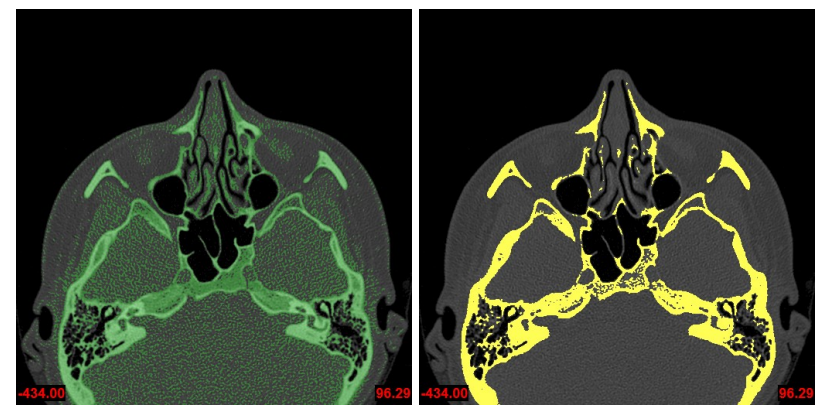

Figure 3. Using the connected area selection function to remove noise on tomograms.

Artifacts can manifest themselves as bands (error in a single measurement), darkening (gradual deviation of a group of channels), rings (calibration errors of a single detector), and distortion (spiral reconstruction). In our case, the patient's mouth contains the objects with a high density, for example, made of metal, which causes the appearance of artifacts in the form of stripes in the image (Fig. 4). This is because the density of the metal is outside the normal range of values that the tomograph can display, giving incomplete attenuation profiles (Chen, 2012) 

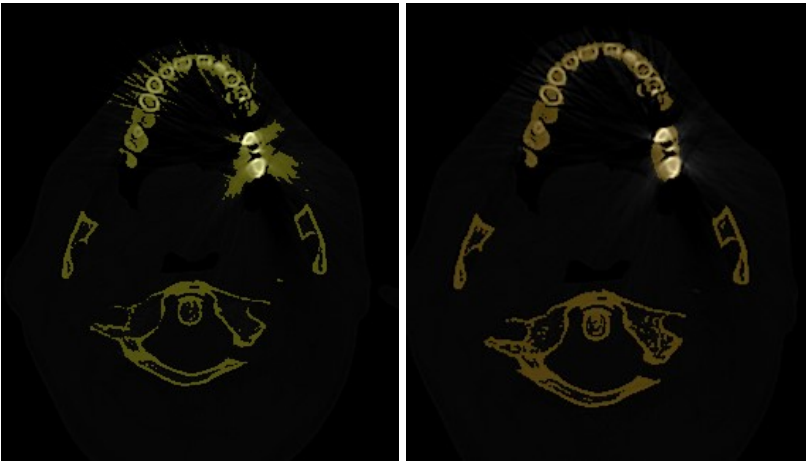

Fig. 4. Manual removal of artifacts caused by the presence of metal.

To eliminate the artifacts caused by the presence of metal, you need to edit each slice separately, removing only unnecessary streaks-glare.

Another criterion for image quality is the contrast resolution of the image - the ability of the scanner to show small changes in the contrast of the tissues of large objects. For a given set of tomograms this setting is important when you are building a thin bone and cartilage of the walls. In the work (Radnionok, 2018), an algorithm for preparing DICOM images for constructing a three-dimensional model of the bones of the facial skull was developed. The DICOM image-processing algorithm reduced the data loss level about the thin bones of the orbit during building a three-dimensional model of the bones of the facial skull from $22-31 \%$ to $3-5 \%$. But it still requires additional processing and manual drawing of fine bone structures.

The figure shows the models built from the source data (on the left) and from the drawn slices with subsequent smoothing (on the right) (Fig. 5).
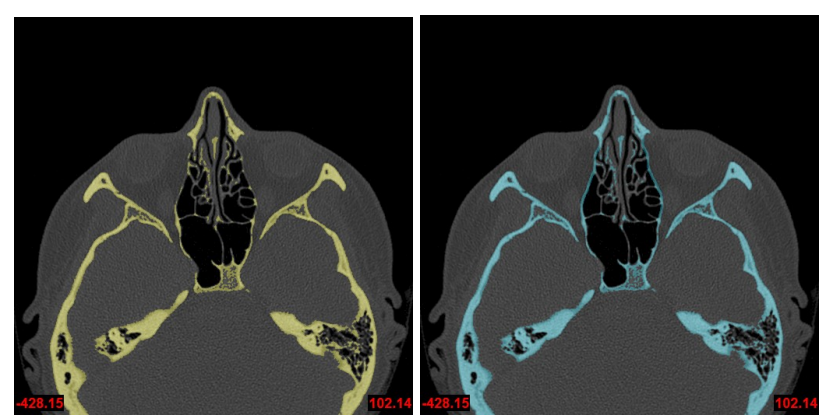

Figure 5. Example of editing tomograms for the restoration of thin bone and cartilage walls and computer models obtained as a result.

The developed methods of processing the tomographic data allow us to build on their basis the computer three-dimensional models of a high degree of accuracy for use in reconstructive surgery and planning of particularly complex operations in neurosurgery.

\section{ISSUES OF CERTIFICATION OF THREE- DIMENSIONAL MODELS FOR MEDICINE}

There are no special standards and certification of digital models in medicine in the Russian Federation today. For the application of digital models, you can use paragraph 5 of Article 38 "Medical devices", Federal Law of November 21, 2011. N 323-FZ "About bases of health protection of citizens in the Russian Federation": "Medical products that are manufactured according to individual orders of the patients, which must meet specific requirements from healthcare workers and are intended solely for the personal use of an individual patient, and medical devices intended for use in the international medical cluster or in the territories of the innovative scientific and technological centres, do not require state registration. These medical devices are not subject to the provisions of part 3 of this article, which provide for the development of technical and (or) operational documentation by the manufacturer (manufacturer) of the medical device."

The US Federal Food and Drug Administration (FDA) issued in 2017 new recommendations for creating medical models using $3 \mathrm{D}$ printers. The recommendations include the sections on the design and manufacturing process, model testing, and drawing up instructions. When creating the models based on the images obtained by CT scanning, the minimum image quality and resolution, image processing algorithms that can change the size of the model compared to real organs, as well as the safety and definability of anatomical landmarks used to adapt the model should be taken into account.

The section on testing the models contains the requirements for their description, results of mechanical tests, measurement of dimensions, material characteristics, sterilization and biocompatibility. Each device must have a manual that specifies the patient's ID, the purpose of the model and its final design, as well as a warning about the need for a preliminary examination of the patient to exclude any changes that may distinguish the model from the actual anatomical structure.

\section{CONCLUSION}

The tools and approaches described in this article are universal and can be used for segmentation of any anatomical structures. In the case of stream diagnostics, the task of segmenting the same organs based on data from the same type of study may be faced many times in a row. In this case, the use of universal tools may not be the fastest and most convenient way of segmentation, and in such tasks it is more effective to use specialized tools (Solovyova, 2018, Thavavel 2020). A specialized segmentation tool can be an implementation of a template for applying universal approaches, or algorithms and methods specially developed for a specific case.

The next task of this work is to develop specialized tools and improve their functionality. The conducted comparative analysis allows us to recommend the use of DICOM Viewer Inobitek and 3DSlicer from the tested programs for processing and converting DICOM images into a three-dimensional model of the STL format.

\section{ACKNOWLEDGMENTS}

This work was done with the financial support of the RFBR (Grant MK 18-29-03238). The work on programs is executed at financial support of the Ministry of science and higher education (State task FSRC "Crystallography and Photonics" RAS). 


\section{REFERENCES}

Chen Yang, Li Yinsheng, Guo Hong, Hu Yining, Luo Limin, Yin Xindao, Gu Jianping, and Toumoulin Christine, 2012: CT Metal Artifact Reduction Method Based on Improved Image Segmentation and Sinogram In-Painting Hindawi Publishing Corporation Mathematical Problems in Engineering Volume, Article ID 786281, 18 pages doi:10.1155/2012/786281

Davies Thomas G. et al. 2017, Open data and digital morphology, Proc. R. Soc. B 284:20170194 http://dx.doi.org/10.1098/rspb.2017.0194

Guang-Ye Wang, Wen-Jun Huang, Qi Song, Yun-Tian Qin \& Jin-Feng Liang, 2016: Computer-assisted virtual preoperative planning in orthopedic surgery for acetabular fractures based on actual computed tomography data, Computer Assisted Surgery, 21:1, 160-165, DOI: 10.1080/24699322.2016.1240235.

Ippolitov E. V., Novikov M. M., Cherebylo S. A., Evseev A.V., 2015: Application of laser stereolithography in medicine Kollektivnaya monografiya: Sovremennye lazernoinformatsionnye tekhnologii. Under the editorship of V. Ya. Panchenko and V. F. Lebedev. Moscow: Interkontakt Nauka, pp. 358-373, ISBN 978-5-902063-52-0.

Mirzoev M. Sh., Shakirov M. N., Khushvakhtov D. I., Dzhonibekova R. N., Grigoriev E. G., 2018: Elimination of mandibular defects by endoprostheses created using additive stereolithographic modeling technology. Bulletin Of Avicenna, 20(2-3):170-175. http://dx.doi.org/10.25005/2074-0581-201820-2-3-170-175.

Maureen van Eijnatten, Juha Koivisto, Kalle Karhu, Tymour Forouzanfar, Jan Wolff, 2017: The impact of manual threshold selection in medical additive manufacturing, J CARS, 12:607615 DOI 10.1007/s11548-016-1490-4.

Musatyan S. A., Lomakin A.V., Sartasov S. Yu., Popyvanov L. K., Monakhov I. B., Chizhova A. S., 2018: Methods of segmentation of medical images. Proceedings of the ISP RAS, volume 30 , issue 4 , pp. 183-194.

Radnionok Anton, Asipovich Vitali, Dudzich Aksana, Krasilnikova Viktoria, Nikolayeu Andrei, Yashin Kanstantsin, 2018: Algorithm of processing microspiral ct-scan results for constructing a three-dimensional model of orbit thin bones, Journal of Engineering Science Vol. XXV, no. 4, pp. 39 - 46.

Solovyova S. N., Urusova V. S., 2018: Development of a model for automatic determination of the boundaries of brain glioma, based on a complex method of processing MRI and CT images. Modern high-tech technologies (RUS) № 5, 145-157

Thavavel Vaiyapuri, Haya Alaskar, 2020: Whale Optimization for Wavelet-Based Unsupervised Medical Image Segmentation: Application to CT and MR Images / International Journal of Computational Intelligence Systems 13(1) 941-953

Trukhan S., Nedzved A., Ablameyko S., 2014: Region growing segmentation of ct-image by the analysis of $3 \mathrm{~d}$ local neighborhood Pattern Recognition and Information Processing (PRIP'2014) : Proceedings of the 12th International Conference (28-30 May 2014, Minsk, Belarus). Minsk, P. 285-289.
Vasilyev S.V., Vasilyeva O.A., Galeev R.M., Dyuzheva O.P., Novikov M. M., Chichaev I.A., Yatsishina E.B., 2019: 3d reconstruction of the ancient egyptian mummy skeleton from the pushkin state museum of fine arts (I,1 1240), The International Archives of the Photogrammetry, Remote Sensing and Spatial Information Sciences, Volume XLII-2/W12, p. 225229, https://doi.org/10.5194/isprs-archives-XLII-2-W12-2252019.

Zesch S. et al. 2016, From First to Latest Imaging Technology: Revisiting the First Mummy Investigated with X-ray in 1896 by Using Dual-Source Computed Tomography // European Journal of Radiology V.3. P. 172. 